\title{
Comunicación
}

\section{Mycoplasma haemominutum en la ciudad de Ibagué (Colombia): reporte de cinco casos}

\author{
Mycoplasma haemominutum in the city of Ibague city \\ (Colombia): report of five cases
}

\author{
Daniel Mayorga ${ }^{1,2}$, Diego Echeverry-Bonilla ${ }^{1,3}$, Edwin Buriticá-Gaviria ${ }^{1}$, \\ Iang Rondón-Barragán ${ }^{2}$
}

\section{Resumen}

\begin{abstract}
El objetivo del presente trabajo fue describir una serie de casos de hemoplasmosis en gatos domésticos en la ciudad de Ibagué (Colombia), y el primer reporte de Mycoplasma haemominutum en el país. Muestras de sangre de pacientes ingresados al servicio de consulta externa de la Clínica de Pequeños Animales de la Universidad del Tolima fueron analizadas por PCR punto final para la amplificación del gen $16 \mathrm{~S}$ ribosomal a fin de obtener un producto final de 194 pb. Además, se hizo biometría hemática y serología para enfermedades virales (Anigen FIV Ab/FeLV Ag Test Kit). Los amplicones obtenidos fueron sometidos a secuenciamiento y análisis bioinformático (Geneious ${ }^{\circledR} 8.1 .9$ ). Se encontró anemia normocítica hipocrómica en dos gatos, leucocitosis en un gato y trombocitopenia en cuatro de ellos. Dos gatos resultaron serorreactivos para anticuerpos de virus de inmunodeficienca felina y antígenos de virus de la leucemia felina (VLFe) y uno solo fue seropositivo a VLFe. Las muestras registraron amplificación positiva en PCR y se evidenció un $98.7 \%$ de identidad al análisis de las secuencias del 16S ribosomal con diferentes aislados de M. haemominutum reportado en la base de datos. Del conocimiento de los autores, este es el primer reporte de hemoplasmas en la ciudad de Ibagué y el primer reporte de $M$. haemominutum en Colombia.
\end{abstract}

Palabras clave: gatos; hemoplasmas; PCR; anemia; virus de inmunodeficiencia felina; virus de leucemia felina

\footnotetext{
${ }^{1}$ Grupo de Investigación en Medicina y Cirugía de Pequeños Animales, Departamento de Sanidad Animal, Facultad de Medicina Veterinaria y Zootecnia, Universidad del Tolima, Colombia

${ }^{2}$ Grupo de Investigación en Inmunobiología y Patogénesis - GIP, Departamento de Sanidad Animal, Facultad de Medicina Veterinaria y Zootecnia, Universidad del Tolima, Colombia

${ }^{3}$ E-mail: decheverry@ut.edu.co
}

Recibido: 2 de diciembre de 2018

Aceptado para publicación: 5 de julio de 2019 
The aim of this study was to describe a series of cases of hemoplasmosis in domestic cats in the city of Ibagué (Colombia), and the first report of Mycoplasma haemominutum in the country. Blood samples from patients admitted to the outpatient service of the Small Animal Clinic of the University of Tolima were analyzed by conventional PCR for the amplification of the $16 \mathrm{~S}$ ribosomal gene to obtain a final product of $194 \mathrm{pb}$. In addition, hematic biometrics and serology were performed for viral diseases (Anigen FIV Ab/FeLV Ag Test kit). The amplicons obtained were subjected to sequencing and bioinformatic analysis (Geneious $\left.{ }^{\circledR} 8.1 .9\right)$. It was found hypochromic normocytic anemia in two cats, leukocytosis in a cat and thrombocytopenia in four of them. Two were seropositive for feline immunodeficiency virus antibodies and feline leukemia virus (FeLV) antigens and one was seropositive for FeLV. Samples showed positive amplification by PCR and revealed $98.7 \%$ of identity of $16 \mathrm{~S}$ ribosomal gene compared with different isolates of $M$. haemominutum reported in the database. From the authors' knowledge, this is the first report of hemoplasmas in the city of Ibague and the first report of M. haemominutum in Colombia.

Key words: cats; haemoplasms; PCR; anaemia; feline immunodeficiency virus; feline leukaemia virus

\section{INTRODUCCIÓN}

Las enfermedades infecciosas de origen bacteriano que afectan a los felinos domésticos son de alta incidencia y están relacionadas con cuadros severos de fiebre, decaimiento y anorexia (Little, 2012). En la casuística de los consultorios veterinarios se describen con frecuencia cuadros clínicos compatibles con micoplamosis felina (Roura et al., 2010), así como en gatos de la calle (Luria et al., 2004), donde son comunes cuadros de anemia, trombocitopenia, pancitopenia y otros signos clínicos no específicos (Barker y Tasker, 2013; Santos et al., 2014).

Tres especies de Mycoplasma han sido implicadas en procesos de enfermedad en el gato doméstico: Mycoplasma haemofelis (Mhf), Candidatus Mycoplasma haemominutum (Mhm) y Candidatus Mycoplasma turicensis (Barker y Tasker, 2013; Tasker et $a l ., 2018)$. La prevalencia de este tipo de infecciones depende de las condiciones am- bientales y geográficas, observándose en la mayoría de los casos infecciones con $\mathrm{Mhm}$ (Assarasakorn et al., 2012; Aquino et al., 2014). El Mhf se ha reconocido como el más patógeno; sin embargo, es frecuente la presencia de coinfecciones con otros hemoplasmas o la presentación concomitante de enfermedades virales como las causadas por el virus de la leucemia felina (VLeF) y el virus de inmunodeficiencia felina (VIF) (Marcondes et al., 2018; Sarvani et al., 2018). En Colombia, el diagnóstico ha estado mayormente limitado a la identificación del microorganismo mediante extendidos de sangre, asociado a la historia clínica y los valores paraclínicos de hematología; mientras que otras técnicas como la serología y la reacción en cadena de la polimerasa (PCR) no se encuentran ampliamente disponibles, de allí que la mayoría de los pacientes reciben desafíos terapéuticos basados en la sospecha clínica.

El presente artículo presenta el primer estudio de casos de micoplasmosis felina en la ciudad de Ibagué utilizando PCR punto final. 


\section{Descripción de los Casos}

Los gatos involucrados en este estudio estuvieron castrados y eran de raza gato común europeo, los cuales fueron referidos a la Clínica de Pequeños Animales de la Universidad del Tolima por presentar algún signo de enfermedad o como parte de una revisión clínica de rutina. Todos ellos tenían acceso de manera libre al exterior de sus viviendas.

\section{Caso 1}

Macho de 8 años, referido por presentar disfagia y pérdida de peso. Al examen clínico se evidenció temperatura rectal de $39.6^{\circ} \mathrm{C}$, frecuencia cardiaca de $140 \mathrm{lpm}$, frecuencia ventilatoria de $52 \mathrm{vpm}$, baja condición corporal (2/5), mucosas ictéricas y deshidratación estimada de $9 \%$. Adicionalmente, se observó enfermedad periodontal tipo III, caracterizada por gingivitis, halitosis y glositis concomitante con un proceso ulceroso.

\section{Caso 2}

Macho de 2 años, referido para examen clínico de rutina. A la evaluación clínica, las constantes fisiológicas fueron normales, temperatura rectal de $38{ }^{\circ} \mathrm{C}$, frecuencia cardiaca de $140 \mathrm{lpm}$, frecuencia ventilatoria de $28 \mathrm{vpm}$, condición corporal (3/5), mucosas rosadas. No se observaron alteraciones clínicas evidentes.

Caso 3

Macho de 1 año, referido por trauma debido a caída de altura. Al examen clínico se evidenció temperatura rectal de $38^{\circ} \mathrm{C}$, frecuencia cardiaca de $116 \mathrm{lpm}$, frecuencia ventilatoria de $64 \mathrm{vpm}$, baja condición corporal $(2 / 5)$, mucosas rosadas. Además, se evidenció enfermedad periodontal tipo I, caracterizada por leve gingivitis, dolor a la palpación y claudicación grado IV de miembro pélvico izquierdo posiblemente asociado a fractura. Adicionalmente, se observó abundante cerumen en conducto auditivo externo y secreción ocular serosa bilateral.

\section{Caso 4}

Macho de 2 años, referido por presentar inflamación del globo ocular derecho. Al examen clínico se evidenció una temperatura de $41{ }^{\circ} \mathrm{C}$, frecuencia cardiaca $196 \mathrm{lpm}$, frecuencia ventilatoria $36 \mathrm{vpm}$, condición corporal (3/5), mucosas rosadas. Así mismo, se observaron laceraciones a nivel del pabellón auricular, enfermedad periodontal con evidencia de gingivitis y descemetocele con fuga de humor acuoso.

\section{Caso 5}

Hembra de 15 años, referida para examen clínico de rutina. Se evidenció temperatura de $38.3{ }^{\circ} \mathrm{C}$, frecuencia cardiaca de 144 $1 \mathrm{pm}$, frecuencia ventilatoria de $53 \mathrm{vpm}$, condición corporal $(2.5 / 5)$ y mucosas rosadas. Así mismo, enfermedad periodontal con presencia de placa, gingivitis y halitosis, seborrea seca y foco alopécico en región cervical.

\section{Pruebas de Laboratorio}

\section{Biometría hemática y serología}

Se obtuvieron muestras sanguíneas de todos los gatos, según metodología descrita por Carvajal (2012). Inicialmente se realizó biometría hemática completa mediante equipo de hematología Hema Screen 18 (AXIOM, Alemania). Posteriormente, se realizaron pruebas SNAP de inmunocromatografía para enfermedades virales (Anigen FIV Ab/FeLV Ag Test Kit, BioNote, Corea del Sur), siguiendo las especificaciones del fabricante.

\section{Pruebas moleculares}

Se extrajo ADN a partir de $200 \mu 1$ de sangre entera utilizando el kit comercial EasyDNA ${ }^{\circledR}$ (Invitrogen, USA), siguiendo las especificaciones del fabricante. La integridad del ADN aislado fue evaluada mediante amplificacion del gen de referencia (constitutivo) beta actina de gato, con un tamaño de 
Cuadro 1. Resultados de las pruebas paraclínicas (biometría hemática, test serológico rápido FIV $\mathrm{Ab} / \mathrm{FeLV} \mathrm{Ag}$, y PCR punto final) aplicadas a cinco gatos comunes europeos en la ciudad de Ibagué (Colombia)

\begin{tabular}{|c|c|c|c|c|c|c|}
\hline \multirow{2}{*}{ Parámetros $^{1}$} & \multicolumn{5}{|c|}{ Casos } & \multirow{2}{*}{$\begin{array}{l}\text { Valores de } \\
\text { referencia }\end{array}$} \\
\hline & 1 & 2 & 3 & 4 & 5 & \\
\hline Eritrocitos, $10^{6} \mu 1$ & 3.36 & 5.96 & 7.71 & 5.43 & 8.68 & $5.0-10.0$ \\
\hline Hemoglobina, g/dl & 5.60 & 6.4 & 9.7 & 7.9 & 11.40 & $8.0-15.0$ \\
\hline Hematocrito, \% & 18.20 & 26.0 & 30.30 & 25.2 & 33.40 & $30-45$ \\
\hline VCM. fl & 54.17 & 43.62 & 30.3 & 46.41 & 38.48 & $39-55$ \\
\hline HCM, pg & 16.67 & 10.74 & 12.58 & 14.55 & 13.13 & $13-17$ \\
\hline CMHC, g/dl & 30.77 & 24.62 & 32.01 & 31.35 & 34.13 & $30-36$ \\
\hline Leucocitos, $10^{3} \mu 1$ & 8.7 & 28.4 & 11.3 & 23.6 & 7.2 & $5.5-19.5$ \\
\hline Neutrófilos segmentados, \% & 76 & 42 & 9 & 70 & 66 & $35-75$ \\
\hline Neutrófilos en banda, $\%$ & 8 & 2 & 0 & 6 & 1 & $0-3$ \\
\hline Eosinófilos, $\%$ & 0 & 10 & 9 & 1 & 7 & $2.0-12.0$ \\
\hline Basófilos, \% & 0 & 0 & 0 & 0 & 0 & - \\
\hline Linfocitos, $\%$ & 15 & 46 & 82 & 22 & 26 & $20-55$ \\
\hline Monocitos, \% & 1 & 0 & 0 & 1 & 0 & $1.0-4.0$ \\
\hline Neutrófilos segmentados, \% & 6.6 & 11.9 & 1 & 16.5 & 4.8 & $2.5-12.5$ \\
\hline Neutrófilos en banda, $10^{3} \mu 1$ & 0.7 & 0.6 & 0 & 1.4 & 0.1 & $0-0.03$ \\
\hline Eosinófilos, $10^{3} \mu 1$ & 0 & 2.8 & 1 & 0.2 & 0.5 & $0-0.75$ \\
\hline Basófilos, $10^{3} \mu 1$ & 0 & 0 & 0 & 0 & 0 & - \\
\hline Linfocitos, $10^{3} \mu 1$ & 1.3 & 13.1 & 9.3 & 5.2 & 1.9 & $1.5-7$ \\
\hline Monocitos, $10^{3} \mu 1$ & 0.1 & 0 & 0 & 0.2 & 0 & $0-085$ \\
\hline Plaquetas, $10^{3} \mu \mathrm{l}$ & 240 & 167 & 220 & 350 & 250 & $300-700$ \\
\hline $\mathrm{Ab}$ FIV,$+/-$ & + & + & - & - & - & - \\
\hline $\mathrm{Ag} \mathrm{FeLV},+/-$ & + & + & - & + & - & - \\
\hline PCR Mycoplasma, +/- & + & + & + & + & + & - \\
\hline
\end{tabular}

amplicón de 353 bp (pares de bases), utilizando los (primers) cebadores (F: CAAGGAGAAGCTCTGCTAC, R: GGTGATCTCCTTCTGCATC) basados en la secuencia reportada en el Genebank (número de acceso XM_006941899.3). La mezcla maestra para el corrido del PCR tuvo un volumen total de $25 \mu \mathrm{l}$ que incluyó $20 \mathrm{mM}$ Tris $\cdot \mathrm{Cl}, 100 \mathrm{mM} \mathrm{KCl}, 1 \mathrm{mM}$ DTT, $0.1 \mathrm{mM}$ EDTA, $200 \mu \mathrm{M}$ de cada dATP, dCTP, y dGTP, $1 \mu \mathrm{M}$ de cada cebador, 0.6 unidades/reacción de Taq polimerasa (TopTaq ${ }^{\mathrm{TM}} \mathrm{PCR}$,
QUIAGEN $^{\mathrm{R}}$ ) y $1 \mu \mathrm{ldel}$ ADN blanco. La amplificación fue realizada con una desnaturalización de $94{ }^{\circ} \mathrm{C}$ durante $3 \mathrm{~min}$, seguidos por 35 ciclos de desnaturalización $\left(94{ }^{\circ} \mathrm{C}, 30 \mathrm{~s}\right)$, anillamiento $\left(55^{\circ} \mathrm{C}, 30 \mathrm{~s}\right)$, extensión $\left(72{ }^{\circ} \mathrm{C}, 30 \mathrm{~s}\right)$ y una extensión final a $72{ }^{\circ} \mathrm{C}$ durante $7 \mathrm{~min}$.

Luego de la evaluación de la integridad del ADN se realizó el PCR para la amplificación del gen 16S ribosomal con el fin de identificar a Mycoplasma (Haemophilus) spp 


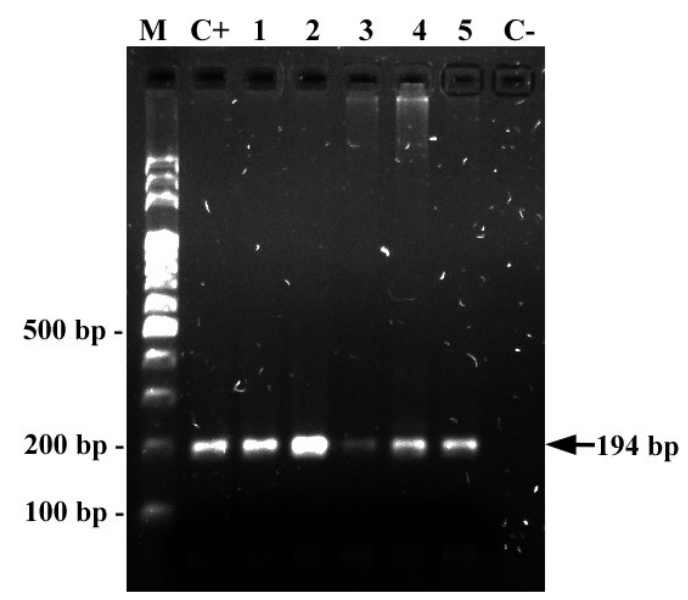

Figura 1. Amplificación del gen 16S ribosomal de Mycoplasma spp a partir de muestras sanguíneas de pacientes felinos. Se observa una banda de aproximadamente $194 \mathrm{bp}$. M: Marcador de peso molecular, $100 \mathrm{bp}$. C+: control positivo. 1-5: Muestras correspondientes a los casos evaluados. C-: control negativo, muestra sin plantilla de ADN. Gel de agarosa al $1 \%$

mediante los cebadores F-ACGAAAGTCTGATGGAGCAATA y R-ATTATTCGGATTTATTGGGCGT, los cuales amplifican un producto de $194 \mathrm{bp}$. Las condiciones de la mezcla maestra fueron iguales a las descritas para la beta actina. Los productos de las reacciones fueron revelados mediante electroforesis horizontal en gel de agarosa al $1 \%$ durante $30 \mathrm{~min}$ a $100 \mathrm{~V}$. La amplificación fue realizada con una desnaturalización de $94{ }^{\circ} \mathrm{C}$ durante $3 \mathrm{~min}$, seguidos por 35 ciclos de desnaturalización $\left(94{ }^{\circ} \mathrm{C}, 30 \mathrm{~s}\right)$, anillamiento $\left(60^{\circ} \mathrm{C}, 30 \mathrm{~s}\right)$, extensión $\left(72^{\circ} \mathrm{C}, 30 \mathrm{~s}\right)$ y una extensión final a $72{ }^{\circ} \mathrm{C}$ durante $7 \mathrm{~min}$. Los amplicones de las muestras positivas fueron purificados y enviados a secuenciamiento por método de Sanger a Macrogen Inc (Seúl, Corea del Sur). La secuencia obtenida fue sometida a análisis bioinformático mediante el software Geneious ${ }^{\circledR}$ 8.1.9 (Biomatters, Nueva Zelanda) (Kearse et al., 2012) usando la base de datos del NCBI y la plataforma BLAST (Basic Local Alignment Search Tool, https:// blast.ncbi.nlm.nih.gov/Blast.cgi).

\section{Resultados}

Los resultados de los parámetros hematológicos y los valores para las pruebas de serología y PCR se presentan en el Cuadro 1 . Se evidenció una anemia regenerativa normocítica e hipocrómica para los pacientes 1 y 2. Leucocitosis con desviación a la izquierda en el paciente 4 y trombocitopenia en los pacientes 1, 2, 3 y 5. Los demás resultados hematológicos no presentaron alteraciones de acuerdo con los rangos de referencia (Merck, 2007).

La prueba SNAP fue positiva para los anticuerpos contra VIF y antígenos para VLeF para los pacientes 1 y 2 . En el paciente 3 solo fueron detectados antígenos para VLeF. Al análisis por PCR, todos los pacientes mostraron la amplificación de una banda de $194 \mathrm{pb}$ (Figura 1). Las secuencias del 16S ribosomal evidenciaron un $98.7 \%$ de identidad con diferentes aislados de Mycoplasma haemominutum $(\mathrm{Mhm})$ reportados en la base de datos.

\section{Discusión}

Díaz-Regañón et al. (2018) reportan una prevalencia de $M h m$ de $0.5 \%$ en gatos de Madrid, España, mientras que MartínezDíaz et al. (2013) reportan prevalencias de hasta $41 \%$ en Portugal. Por otro lado, en América del Sur se han reportado prevalencias de $7.8 \%$ en Chile (Walker et al., 2016) hasta de $11.2 \%$ en Brasil (Santis et al., 2014). En Colombia solo se han reportado casos de hemoplasmosis felina como diagnóstico presuntivo basados únicamente en los hallazgos clínicos, extendidos de sangre y valores hematológicos (Ortiz, 2011). Del conocimiento de los autores, solo ha sido reportado un estudio empleando técnicas 
moleculares, utilizando PCR convencional para el diagnóstico de hemoplasmas en muestras de 420 gatos de dos refugios para animales localizados en las ciudades de Calí y Bogotá, encontrándose $97 \%$ de frecuencia de presentación de Mycoplasma haemofelis (Carvajal, 2012). El presente estudio describe por primera vez el diagnóstico molecular de hemoplasmas en la ciudad de Ibagué y de $M h m$ en el país utilizando PCR punto final.

Diversos estudios han demostrado la correlación entre el número de células rojas, el bajo hematocrito y la positividad a hemoplasmas (Ghazisaeedi et al., 2014; Hwang et al., 2015). Para los casos 1, 2 y 4 fue evidente una disminución de estos parámetros; sin embargo, otros trabajos no han evidenciado dicha correlación entre la infección por Mhm y la presentación de anemia (Aquino et al., 2014; Santos et al., 2014; Sarvani et al., 2018), por lo que es posible que otros factores, como la coinfección con enfermedades virales, puedan influir en la variación de valores hematológicos (Shelton et al., 1990; Gleich y Hartmann, 2009; Hartmann, 2011; da Costa et al., 2017). En el presente trabajo, la prueba de inmunocromatografía positiva para antígenos de LeVF en los pacientes 1,2 y 4 no permite esclarecer la fase de la infección y, por lo tanto, es posible que la presentación de anemia esté sujeta a otras patologías no establecidas.

Firmino et al. (2016) encontraron una disminución en el número de glóbulos blan$\cos$, neutrófilos segmentados y plaquetas en los gatos con coinfección de Mhm y LeVF. En el presente estudio, los pacientes 1 y 2 presentaron esta coinfección junto con una disminución en el conteo plaquetario; sin embargo, el número de leucocitos aumentó en el paciente 2; así mismo, la disminución en el conteo plaquetario fue evidente en los pacientes 3 y 5. Ghazisaeedi et al. (2014), por otro lado, encontraron una disminución en el conteo plaquetario en pacientes infectados con hemoplasmas, pero otras causas de trombocitopenia pudieran explicar igualmente los resultados.
Algunos estudios demuestran una asociación entre la infección por hemoplasmas con animales con acceso al exterior y con parásitos externos, especialmente pulgas (Spada et al., 2014) lo cual puede indicar una transmisión directa por picadura (Woods et al., 2005) o por mordeduras de otros gatos (Dean et al., 2008). En los pacientes del presente estudio no se evidenciaron parásitos externos, por lo cual es difícil establecer su presencia como posible mecanismo de infección. Por otro lado, ser macho, mestizo o positivo a FIV son factores de riesgo asociados a la presencia de hemoplasmas (Jenkins et al., 2013; Rosenqvist et al., 2016; Walker et al., 2016; Attipa et al., 2017) y a la infección con $M h f$ (Sarvani et al., 2018); sin embargo, hay trabajos que solo describen la coinfección con FIV como único factor de riesgo para Mhf (Bauer et al., 2008). En el presente estudio, los cinco pacientes fueron mestizos, 4 fueron machos y 2 fueron resultaron positivos a antígenos contra VIF. Así mismo, Sarvani et al. (2018) demostraron que el acceso a la calle aumenta 5.54 veces la probabilidad de infección con $M h f$, característica que fue un común denominador en los cinco casos de este estudio.

Múltiples estudios han evaluado la presencia de $M h m$, tanto en gatos sanos como enfermos, no hallándose una clara asociación entre la infección y el desarrollo de la enfermedad en gatos inmunocompetentes (Barker y Tasker, 2013; Ghazisaeedi et al., 2017).

El presente reporte evidencia por primera vez la presencia de $M h m$ en el país y la presencia de hemoplasmas en la ciudad de Ibagué. Es importante que los médicos veterinarios tanto de Ibagué como de Colombia reconozcan la presencia potencial de $\mathrm{Mhm}$ en los gatos domésticos, y adopten medidas para su diagnóstico y tratamiento. Estudios adicionales son necesarios para establecer la prevalencia de hemoplasmas y su impacto sobre la salud animal. 


\section{Agradecimientos}

Este proyecto de investigación fue financiado con recursos del Fondo de Ciencia, Tecnología e Innovación del Sistema General de Regalías de la República de Colombia (Proyecto Código 420120516). Los autores agradecen a la Oficina Central de Investigaciones y Desarrollo Científico de la Universidad del Tolima por su apoyo en la realización de este estudio.

\section{Literatura Citada}

1. Aquino LC, Hicks CAE, Scalon MC, Lima MG, Lemos M, Paludo G, Helps $C$, et al. 2014. Prevalence and phylogenetic analysis of haemoplasmas from cats infected with multiple species. J Microbiol Meth 107: 189-196.

2. Assarasakorn S, Veir JK, Hawley JR, Brewer MM, Morris AK, Hill AE, Lappin MR. 2012. Prevalence of Bartonella species, hemoplasmas, and Rickettsia felis DNA in blood and fleas of cats in Bangkok, Thailand. Res Vet Sci 93: 1213-1216. doi: 10.1016/ j.rvsc.2012.03.015

3. Attipa C, Papasouliotis K, Solano-Gallego L, Baneth G, Nachum-Biala Y, Sarvani E, Knowles TG. 2017. Prevalence study and risk factor analysis of selected bacterial, protozoal and viral, including vector-borne, pathogens in cats from Cyprus. Parasite Vector 10: 130. doi: 10.1186/s13071-017-2063-2

4. Barker E, Tasker S. 2013. Haemoplasmas: lessons learnt from cats. New Zeal Vet J 61: 184-192. doi: 10.1080/ 00480169.2013.771760

5. Bauer N, Balzer HJ, Thüre S, Moritz A. 2008. Prevalence of feline haemotropic mycoplasmas in convenience samples of cats in Germany. J Feline Med Surg 10: 252-258. doi: 10.1016/ j.jfms.2007.12.004

6. Carvajal D. 2012. Frecuencia de infecciones rickettsiales y hemoparasitarias en gatos domésticos (Felis catus
Schereber 1775) de los Centros de Zoonosis, en las Ciudades de Bogotá y Cali. Tesis de Maestría. Colombia: Univ. Nacional de Colombia. $138 \mathrm{p}$.

7. da Costa FV, Valle S, Machado G, Corbellini L, Coelho E, Rosa RB, González FH. 2017. Hematological findings and factors associated with feline leukemia virus (FeLV) and feline immunodeficiency virus (FIV) positivity in cats from southern Brazil. Pesquisa Vet Brasil 37: 1531-1536. doi: 10.1590/ s0100-736x2017001200028

8. Dean RS, Helps CR, Gruffydd Jones TJ, Tasker S. 2008. Use of real-time PCR to detect Mycoplasma haemofelis and «Candidatus Mycoplasma haemominutum» in the saliva and salivary glands of haemoplasma-infected cats. J Feline Med Surg 10: 413-417. doi: 10.1016/j.jfms.2007.12.007

9. Díaz-Regañón D, Villaescusa A, Ayllón T, Rodríguez-Franco $F$, García-Sancho M, Agulla B, Sainz Á. 2018. Epidemiological study of hemotropic mycoplasmas (hemoplasmas) in cats from central Spain. Parasite Vector 11: 140. doi: 10.1186/s13071-0182740-9

10. Firmino FP, Aquino LC, Marçola TG, Bittencourt MV, McManus CM, Paludo GR. 2016. Frequency and hematological alterations of different hemoplasma infections with retrovirusis co-infections in domestic cats from Brazil. Pesquisa Vet Brasil 36: 731-736. doi: 10.1590/S0100-736X2016000800009

11. Ghazisaeedi F, Atyabi N, Zahrai T, Gentilini F, Ashrafi I, Akbarein H, Tasker S. 2014. A molecular study of hemotropic mycoplasmas (hemoplasmas) in cats in Iran. Vet Clin Path 43: 381-386. doi: 10.1111/vep. 12166

12. Gleich S, Hartmann K. 2009. Hematology and serum biochemistry of feline immunodeficiency virus-infected and feline leukemia virus-infected cats. J Vet Intern Med 23: 552-558. doi: 10.1111/ j.1939-1676.2009.0303.x 
13. Hartmann K. 2011. Clinical aspects of feline immunodeficiency and feline leukemia virus infection. Vet Immunol Immunop 143: 190-201. doi: 10.1016/ j.vetimm.2011.06.003

14. Hwang J, Gottdenker N, Min MS, Lee H, Chun MS. 2015. Evaluation of biochemical and haematological parameters and prevalence of selected pathogens in feral cats from urban and rural habitats in South Korea. J Feline Med Surg 18: 443-451. doi: 10.1177/ 1098612X15587572

15. Jenkins KS, Dittmer KE, Marshall JC, Tasker S. 2013. Prevalence and risk factor analysis of feline haemoplasma infection in New Zealand domestic cats using a real-time PCR assay. J Feline Med Surg 15: 1063-1069. doi: 10.1177/ 1098612X13488384

16. Kearse M, Moir R, Wilson A, StonesHavas S, Cheung M, Sturrock $S$, Buxton S, et al. 2012. Geneious basic: an integrated and extendable desktop software platform for the organization and analysis of sequence data. Bioinformatics 28: 1647-1649. doi: 10.1093/bioinformatics/bts 19

17. Little SE. 2012. The cat: clinical medicine and management. St. Louis: Elsevier. $1424 \mathrm{p}$.

18. Lobetti RG, Tasker S. 2004. Diagnosis of feline haemoplasma infection using a real-time PCR assay. J S Afr Vet Asso 75: 94-99. doi: 10.4102/jsava.v75i2.460

19. Luria BJ1, Levy JK, Lappin MR, Breitschwerdt EB, Legendre AM, Hernandez JA, Gorman SP, et al. 2004. Prevalence of infectious diseases in feral cats in Northern Florida. J Feline Med Surg 6: 287-296. doi: 10.1016/ j.jfms.2003.11.005

20. Marcondes M, Hirata KY, Vides JP, Sobrinho LSV, Azevedo JS, Vieira TSWJ, Vieira RFC 2018. Infection by Mycoplasma spp, feline immunodeficiency virus and feline leukemia virus in cats from an area endemic for visceral leishmaniasis. Parasite Vector 11: 131. doi: 10.1186/s13071-018-2716-9

21. Martínez-Díaz VL, Silvestre-Ferreira AC, Vilhena H, Pastor J, Francino O, Altet L. 2013. Prevalence and coinfection of haemotropic mycoplasmas in Portuguese cats by real-time polymerase chain reaction. J Feline Med Surg 15: 879-885. doi: 10.1177/1098612X13480985

22. Merck. 2007. The Merck veterinary manual. $11^{\text {th }}$ ed. Merck Manuals.

23. Ortiz JF. 2011. Three clinical cases of feline leukemia associated with regenerative anemia, breast carcinoma, or peritonitis. Rev Colom Cienc Pecua 24: 55-62.

24. Rosenquist MB, Meilstrup AKH, Larsen J, OlsenJE, Jensen $A L$, Thomsen LE. 2016. Prevalence of feline haemoplasma in cats in Denmark. Acta Vet Scand 58: 78. doi: 10.1186/ s13028-016-0260-1

25. Roura X, Peters IR, Altet L, Tabar M, Barker EN, Planellas M., Helps C, et al. 2010. Prevalence of hemotropic mycoplasmas in healthy and unhealthy cats and dogs in Spain. J Vet Diagn Invest 22: 270-274. doi: 10.1177/104063871002200219

26. Santis G, Miraglia H, Marques KC, Gonçalves LR, Denardi N, Domingos I, Campos JB, et al. 2014. Molecular detection of hemotrophic mycoplasmas among domiciled and free-roaming cats in Campo Grande, state of Mato Grosso do Sul, Brazil. Rev Bras Parasitol V 2961: 231-236. doi: 10.1590/S198429612014039

27. Santos AP, Conrado FO, Messick JB, Biondo AW Oliveira ST2, Guimaraes AM, Nascimento NC, et al. 2014. Hemoplasma prevalence and hematological abnormalities associated with infection in three different cat populations from Southern Brazil. Rev Bras Parasitol V 23: 428-434. doi: 10.1590/S198429612014079 
28. Sarvani E, Tasker S, KovacjeviaeFilipovice M, Francuski-Andrice J, Andrice N, Aquino L, English S, et al. 2018. Prevalence and risk factor analysis for feline haemoplasmas in cats from Northern Serbia, with molecular subtyping of feline immunodeficiency virus. JFMS Open Rep 4: 2055116918770037. doi: 10.1177/2055116918770037

29. Shelton GH1, Linenberger ML, Grant CK, Abkowitz JL. 1990. Hematologic manifestations of feline immunode-ficiency virus infection. Blood 76: 1104-1109.

30. Spada E, Proverbio D, Galluzzo P, Della A, Bagnagatti G, Perego $R, F e-$ rro E. 2014. Prevalence of Haemoplasma infections in stray cats in northern Italy. ISRN Microbiol 2014: 298352.

31. Tasker S, Hofmann-Lehmann R, Belák S, Frymus T, Addie DD, Pennisi MG, Boucraut-Baralon C, et al. 2018. Haemoplasmosis in cats: European guidelines from the $\mathrm{ABCD}$ on prevention and management. J Feline Med Surg 20: 256-261. doi: 10.1177/1098612X18758594.

32. Walker-Vergara $R, \quad M o r e r a-$ Galleguillos F, Gómez-Jaramillo M, Pereira-Almosny NR, AraunaMartínez, P, Grob-Behne P, AcostaJamett GA. 2016. Prevalence, risk factor analysis, and hematological findings of hemoplasma infection in domestic cats from Valdivia, Southern Chile. Comp Immunol Microb 46: 20-26. doi: 10.1016/ j.cimid.2016.03.004

33. Woods JE, Brewer MM, Hawley JR, Wisnewski N, Lappin MR. 2005. Evaluation of experimental transmission of Candidatus Mycoplasma haemominutum and Mycoplasma haemofelis by Ctenocephalides felis to cats. Am J Vet Res 66: 1008-1012. doi: 10.2460/ ajvr.2005.66.1008 